AperTO - Archivio Istituzionale Open Access dell'Università di Torino

\title{
Antiphospholipid Syndrome and the Kidney
}

\section{This is the author's manuscript}

Original Citation:

Availability:

This version is available http://hdl.handle.net/2318/1546028

since 2016-10-20T16:26:54Z

Published version:

DOI:10.1016/j.semnephrol.2015.08.009

Terms of use:

Open Access

Anyone can freely access the full text of works made available as "Open Access". Works made available under a Creative Commons license can be used according to the terms and conditions of said license. Use of all other works requires consent of the right holder (author or publisher) if not exempted from copyright protection by the applicable law. 
This Accepted Author Manuscript (AAM) is copyrighted and published by Elsevier. It is posted here by agreement between Elsevier and the University of Turin. Changes resulting from the publishing process - such as editing, corrections, structural formatting, and other quality control mechanisms - may not be reflected in this version of the text. The definitive version of the text was subsequently published in SEMINARS IN NEPHROLOGY, 35 (5), 2015, 10.1016/j.semnephrol.2015.08.009.

You may download, copy and otherwise use the AAM for non-commercial purposes provided that your license is limited by the following restrictions:

(1) You may use this AAM for non-commercial purposes only under the terms of the CC-BY-NC-ND license.

(2) The integrity of the work and identification of the author, copyright owner, and publisher must be preserved in any copy.

(3) You must attribute this AAM in the following format: Creative Commons BY-NC-ND license (http://creativecommons.org/licenses/by-nc-nd/4.0/deed.en), 10.1016/j.semnephrol.2015.08.009

The publisher's version is available at:

http://linkinghub.elsevier.com/retrieve/pii/S0270929515001473

When citing, please refer to the published version.

Link to this full text:

http://hdl.handle.net/2318/1546028 


\section{Antiphospholipid Syndrome and the Kidney}

Savino Sciascia, MD, PhD, ${ }^{*},+$ Simone Baldovino, MD, ${ }^{*}$ Karen Schreiber, MD, MRCP, $\dagger, \ddagger$ Laura Solfietti, BSc, ${ }^{*}$ and Dario Roccatello, MD*,§

Summary: The antiphospholipid syndrome (APS) is a systemic autoimmune disorder characterized by a combination of arterial and/or venous thrombosis, pregnancy morbidity, and the persistent presence of circulating antiphospholipid antibodies (aPL). APS has been described as primary APS when it occurs in the absence of any features of other autoimmune disease, and as secondary in the presence of other autoimmune diseases, mainly systemic lupus erythematosus (SLE). There is a well-known link between SLE and APS; $40 \%$ of SLE patients have aPL, and, in turn, some, but only a minority of patients with APS, eventually will develop features of SLE. Because SLE and APS can target the kidneys independently or at the same time, discriminating between inflammatory or thrombotic lesions is crucial in planning therapy. We provide an overview of the renal manifestations associated with the presence of aPL in patients with SLE, and discuss the impact of aPL in selected scenarios such as lupus nephritis, end-stage renal disease, and pregnancy.

Keywords: antiphospholipid antibodies, thrombosis, antiphospholipid syndrome, APS nephropathy

* Center of Research of Immunopathology and Rare Diseases, Department of Clinical and Biological Sciences, University of Turin, Turin, Italy. † Louise Coote Lupus Unit, Guy's and St Thomas' National Health Service (NHS) Foundation Trust, St Thomas' Hospital, London, UK. ¥ Department of Rheumatology, University Hospital at Rigshospitalet, Copenhagen. § Nephrology and Dialysis Unit, Department of Medicine, San Giovanni Bosco Hospital, Turin, Italy

The antiphospholipid syndrome (APS) is a systemic autoimmune disorder characterized by a combination of arterial and/or venous thrombosis and the persistent presence of circulating antiphospholipid antibodies (aPL).1 APS has been described as primary APS when it occurs in the absence of any features of other autoimmune disease, and as secondary in the presence of other autoimmune diseases, mainly systemic lupus erythematosus (SLE). Because SLE and APS can target the kidneys independently or at the same time, discriminating between inflammatory and thrombotic lesions is crucial in planning an adequate therapeutic strategy. The incidence of kidney involvement in SLE ranges from $30 \%$ to $50 \%$, depending on the definition of abnormal renal function, and represents a severe organ manifestation that is associated with significant patient morbidity and mortality.2,3 Although all renal compartments (glomerular, tubulointerstitial, and vascular) can be injured in SLE, the term lupus nephritis refers specifi- cally to immune-complex mediated glomerulonephritis. Lupus nephritis (LN) presents with diverse clinical and histologic manifestations. However, the main driver of $L N$ remains the immune system and therefore the main therapeutic approach is immunosuppression. 4 Among SLE patients, the prevalence of aPL ranges from $30 \%$ to $40 \% .5$ Approximately $50 \%$ of patients with both SLE and aPL will develop APS during longterm (10-20 y) follow-up evaluation. 5 The presence of aPL in patients with SLE and LN has been shown to be associated with increased mortality and a poorer prognosis, and renal APS requires treatment beyond immunosuppression. 6 In this review we describe the role of aPL/APS in kidney injury in SLE, and focuses on state-of-the art treatment and future therapies of APS. aPL SPECIFICITIES AND THROMBOSIS APS has been known for over three decades and advances in research have identified the coagulation system and the immune system as key players underlying the clinical manifestations of the syndrome. 5 The persistent presence of aPL is crucial for the classification of APS. aPL are recognized as a heterogeneous group of 
antibodies directed against anionic phospholipids or protein-phospholipid complexes. Laboratory tests aimed to identify aPL include solid-phase immunoassays (enzyme-linked immunosorbent assay) to detect anticardiolipin ( $\mathrm{aCL}$ ) and anti- $\beta 2$ glycoprotein 1 (aß2GPI) antibodies, and functional assays to detect lupus anticoagulant (LAC). LAC has the ability to interfere with phospholipid-dependent coagulation reactions. The first international consensus statement on classifi- cation criteria for APS was developed in 1998 in Sapporo, Japan. Eight years later the consensus criteria were updated.1 According to the current classification criteria of APS (Table 1), at least one clinical manifestation is needed. This may include any vascular thrombosis and/or pregnancy morbidity in combination with a positive laboratory test, including $\mathrm{LAC}, \mathrm{aCL}$, or a $32 \mathrm{GPI}$, detected at least twice 12 weeks apart. The syndrome can present with a variety of clinical manifestations caused by thrombosis of any vessel. Deep vein thrombosis and pulmonary embolism are the most frequent features in the venous vasculature. 6 The most common arterial manifestations are neurologic, such as transient ischemic attacks or strokes, or dermatologic manifestations, such as livedo reticularis, seen in $20 \%$ of APS patients. aPL-mediated placental dysfunction, which partially, but not entirely, can be explained by thrombosis, may cause intrauterine growth restriction, preeclampsia, intrauterine fetal death, or placental abruption. 7 Thromboses are the main cause of renal involvement in APS and can occur at any level within the renal vasculature. These thrombotic events can lead to various manifestations that reflect the site and size of the involved vessel. A substantial body of evidence supports the inclusion of aPL-related nephropathy in the classification criteria for definitive APS.8 aPL ANTIBODIES AND THROMBOSIS IN VIVO A two-hit hypothesis for the pathogenesis of APS has been suggested. This is based on the fact that the presence of aPL in the circulation sometimes, but not always, leads to thrombotic events. Therefore, the presence of aPL is necessary but not sufficient for thrombi formation in vivo. This hypothesis implies that the presence of aPL (the first hit) does not itself cause a thrombus, but increses the risk of any thrombotic events when another prothrombotic factor is added (the second hit).9,10 Thus, according to this model, the presence of aPL creates a prothrombotic state, while the second hit leads to thrombus formation. In this regard, it is useful to speculate on the reported association between an acute thrombotic event and a recent or concomitant infectious process in APS patients, or with a traumatic or inflammatory stimulus in experimental models.11 Furthermore, a genetically determined thrombophilic diathesis, or ability to mount a prompt inflammatory response, may enhance the thrombotic risk of subjects with aPL further. The proposed pathophysiological mechanisms underlying aPL-mediated thrombosis in APS have been divided into two main types.10,12 First, aPL may act in vivo by disrupting the normal balance between procoagulants and anticoagulants. Second, aPL may interact with specific cell surface receptors (proteins and/or lipids), inducing downstream signals that up-regulate procoagulant cell surface proteins.13

For example, it appears that the expression of tissue factor, the main activator of the coagulation system, is increased on endothelial cells and monocytes in APS.13 aPL specifically affects platelets, endothelial cells, and monocytes, at least in part through binding to $\beta 2 \mathrm{GPI}$ expressed on cell membranes.13 aPL also affects plasma coagulation regulatory proteins, coagulation cascade components (such as prothrombin, antithrombin, proteins $\mathrm{C}$ and $\mathrm{S}$, factor $\mathrm{X}$ ), and fibrinolysis.10,13 Other processes suggested to play a role in aPLinduced thrombosis include conformational and posttranslational redox modifications of $\beta 2 \mathrm{GPI}$, decreased activity of endothelial nitric oxide synthase, and complement activation. Traditional cardiovascular risk factors also may contribute to the aPL-induced prothrombotic state and are found in more than $50 \%$ of patients with APS. These risk factors are of particular relevance given their modifiable nature. We recently reported that arterial hypertension and hyperlipidemia, together with different aPL specificities, are independent risk factors for thrombotic events in APS.14 APS AND RENAL MANIFESTATIONS As mentioned previously, APS is an autoimmune disease characterized by the presence 
of aPL, arterial or venous thrombosis, and/or pregnancy complications. Any organ can be affected in this disorder; thus, the range of clinical features is extremely wide (Table 2).

Vessels of all sizes may be affected by thrombus formation, and the vascular pathologic appearance consistently has been of bland occlusion without inflammatory infiltrates in the vessel walls.15 Amigo et al16 first described renal involvement in APS in 1992. Since then, a spectrum of renal thrombotic manifestations has been described in association with aPL, such as renal artery stenosis, renal infarction, renal vein thrombosis, and acute or chronic thrombotic microangiopathy (TMA)17,18 (Table 3). A distinct clinical entity, the so-called, "aPL nephropathy," has been described.19,20 The clinical and histologic manifestations of aPL nephropathy reflect the renal vessels affected and include ischemic glomeruli and thrombotic lesions, without glomerular or arterial immune deposits on immunofluorescence.21 APS NEPHROPATHY The term APS nephropathy (APSN) refers to renal damage caused by vascular lesions in the glomeruli, arterioles, and/or interlobular arteries in patients with persistently present aPL. APSN has been described in aPL carriers, in patients with primary APS, and in patients with secondary APS (especially in patients with SLE), in whom it can coincide with the presence of LN.22 APSN lesions can present as acute lesions (so-called TMA) and/or chronic arteriosclerosis, tubular thyroidization, fibrous intimal hyperplasia, and focal cortical atrophy.16,23

Clinical Features APSN may manifest with a nephritic or nephrotic picture, acute or chronic renal failure, hypertension, hematuria, and (mostly mild) proteinuria.16,19 The diagnosis of APSN can be a particular challenge in patients with underlying SLE, and should be supported by a kidney biopsy. Differential Diagnosis When confronted with a clinical picture of systemic or intrarenal platelet aggregation and thrombocytopenia in combination with microvascular fragmentation of erythrocytes resulting in ischemic end-organ injury, the main differential diagnosed in addition to APS include other causes of TMA, such as thrombotic thrombocytopenic purpura or hemolytic uremic syndrome.23 Severe thrombocytopenia and anemia are observed more commonly in thrombotic thrombocytopenic purpura/hemolytic uremic syndrome and the presence of peripheral blood schistocytes rarely is observed in APS. A useful tool to distinguish between APS and other possible causes of TMA is a test for aPL.24 The clinical utility of aPL assays for autoantibodies other than those assessed routinely, such as aCL and a $32 \mathrm{GPI}$, is under debate. 25 Indeed, current lines of research are examining the usefulness of testing for new aPL specificities (such as antiprothrombin antibodies) in helping to identify APS in patients with thrombosis and/or pregnancy morbidity, particularly in patients who repeatedly are negative for the currently used tests. 25 Catastrophic APS (CAPS) is a particularly severe form of APS, and the kidney is the most commonly involved organ. A definitive diagnosis of CAPS requires the following: (1) clinical evidence of involvement of three or more organ systems in a period of less than a week, (2) histopathologic evidence of small-vessel occlusion in at least one organ system, and (3) laboratory confirmation of the presence of aPL, usually in high titers.26 CAPS is associated with a high mortality rate and is therefore an important differential to consider. 26

Histologic Findings Histologically, vaso-occlusive lesions caused by acute microthrombi and chronic vascular lesions including intimal hyperplasia, fibrous occlusions with focal atrophy, and recanalization of occluded vessels can be found27 (Fig. 1). Ultrastructural changes may show the co-existence of glomerular basement membrane reduplication and glomerular basement membrane wrinkling.16 TMA is a common distinct histologic finding in APSN, although it is not an exclusive finding in APS and not all patients present with a full picture of TMA. The formation of glomerular microthrombi has also been described in connection with aPL.28,29 Among patients with SLE and diffuse proliferative glomerulonephritis, up to $20 \%$ to $30 \%$ were shown to have glomerular microthrombi, and they have been described as a strong predictor of glomerular sclerosis and subsequent poor renal outcome.29,30 This raises the question of whether microthrombi 
formation can be regarded as part of the TMA spectrum. Zheng et al29 reported an association of the presence of glomerular microthrombi with detection of LAC and aß2GPI in SLE patients. In the cohort of $124 \mathrm{LN}$ patients studied by Zheng et al,29 serum complement C3 levels were lower, and C3 and C1q staining was more intense compared with patients without glomerular microthrombi. In a retrospective analysis of 114 renal biopsy specimens from lupus patients, Daugas et al19 showed that APSN occurred in 32\% of patients in addition to and independently from lupus nephritis. APSN statistically was associated with aPL, mainly LAC and with extrarenal manifestations of APS, such as arterial thrombosis and obstetric fetal loss. In their cohort, no association between APSN and venous thrombosis was found. The investigators concluded that APSN was an independent risk factor favoring an increased prevalence of hypertension, an increased serum creatinine level, and an increased prevalence of interstitial fibrosis in SLE.19 Tektonidou et al18 studied the prevalence, clinical associations, and long-term outcomes of APSN. APSN was documented in approximately two thirds of SLE patients with secondary APS. The investigators concluded that LAC and/or aCL was associated with TMA, but not with LN, and hence that APS nephropathy occurred almost exclusively in patients with aPL, confirming the important role of aPL in the pathogenesis of APS nephropathy. In line with Daugas et al,19 they found an association with hypertension, increased serum creatinine levels, and histologic progression, all of which were associated with a poor renal outcome. aPL in Lupus Nephritis APS contributes significantly to the renal morbidity in patients with lupus nephritis.19,31 Moroni et al32 found that the presence of aPL in LN patients increased the risk of vascular and obstetric complications, and showed a strong association between aPL antibodies and prognosis in patients with LN. In their cohort of 111 patients, aPL antibody positivity, high plasma creatinine level at presentation, and chronicity index were independent predictors of chronic renal function deterioration. These results are in line with Pérez Velásquez et al,33 who examined a cohort of 600 patients with SLE. This group found not only that LAC and anticardiolipin were more prevalent, but also that more than half of patients with secondary APS had renal disease (56\%) and $43 \%$ showed typical features of APS on renal biopsy. The significance of APSN is reflected in the longterm outcome of these patients. Tektonidou et al18 studied SLE patients (with or without $\mathrm{aPL}$ ) to determine the prevalence and long-term outcome of APSN, as well as the histologic evolution of APSN lesions on serial kidney biopsy specimens. In their cohort of 151 patients, APSN was documented, in addition to and independently from lupus nephritis, in almost $40 \%$ of patients with aPL compared with only 3 of 70 (2.1\%) patients without aPL, suggesting a critical role of aPL in the pathogenesis of APSN. Compared with patients without APSN, patients with APSN had a higher frequency of hypertension and increased serum creatinine levels at the time of kidney biopsy, and developed progression of histologic lesions, all of which were associated with a poor renal outcome.18 SLE, APS, and Pregnancy SLE commonly affects women during their childbearing years, and pregnancy is therefore an important topic. SLE has a tendency to flare during pregnancy and consequently may impact renal function with possible long-term consequences such as end-stage renal disease. Pregnancy counseling to ensure disease remission, close follow-up evaluation during pregnancy, and post-partum surveillance play a major role in improving pregnancy outcomes.34 The presence of aPL is a well-documented risk factor for pregnancy morbidity, including recurrent abortions, premature delivery, intrauterine growth restriction, and preeclampsia, and can be found in $25 \%$ of SLE pregnancies. 35 Post-partum renal failure in association with the presence of aPL was described in the 1980s by Kincaid-Smith et al.36,37 A recent metaanalysis by Smyth et al35 confirmed a positive association between the presence of aPL and hypertension in pregnancy, premature birth, and recurrent miscarriages. A cohort of 1842 patients and 2751 pregnancies was examined. Maternal complications included lupus flare (25.6\%), hypertension (16.3\%), nephritis (16.1\%), preeclampsia (7.6\%), and eclampsia (0.8\%). Fetal complications included spontaneous abortion $(16.0 \%)$, stillbirth (3.6\%), neonatal deaths (2.5\%), and intrauterine growth restriction (12.7\%), with a premature birth rate of $39.4 \% .35$ Treatment The treatment of asymptomatic aPL carriers without 
underlying autoimmune disease remains controversial. However, a recent consensus document emphasized that there is no role for primary thromboprophylaxis with low-titer aPL or intermittent aPL positivity (Table 4).38 In asymptomatic aPL carriers with underlying autoimmune conditions, especially SLE, primary thromboprophylaxis should be considered because this condition itself may be thought of as an additional risk factor for thrombosis. Therefore, primary thromboprophylaxis with low-dose aspirin (75-100 $\mathrm{mg} /$ day) could be considered in all patients with an underlying systemic autoimmune condition and persistent aPL at medium-high titers (IgM or IgG phospholipid units $440 \mathrm{GPL}$ or MPL or 4 99th percentile). In patients with SLE specifically and persistently positive aPL, primary thromboprophylaxis including lowdose aspirin (75- $100 \mathrm{mg} /$ day) and/or hydroxychloroquine (200-400 mg/day) is recommended. This suggestion is based on retrospective studies that have shown that hydroxychloroquine appears to protect SLE patients, especially those with aPL, against thrombosis.39 Although no study has investigated specifically whether the addition of antiplatelet agents offers additional protection, aspirin also may be considered in the setting of primary thromboprophylaxis. 40 Given the general recommendation of hydroxychloroquine administration in most patients with SLE, the addition of low-dose aspirin should be decided on an individual basis. The addition of lowdose aspirin may be appropriate in selected cases, such as patients with a high-risk aPL profile (eg, triple positivity for lupus anticoagulant, aCL and aß2GPI), other concomitant cardiovascular risk factors, and/or SLE patients with a history of obstetric APS. In patients with previous thrombosis (regardless of any underlying autoimmune disease) anticoagulation with a vitamin $\mathrm{K}$ antagonist (international normalized ratio, 2.0-3.0) is required as long-term secondary thromboprophylaxis.41,42 Patients with arterial events or cases of recurrent thrombotic events despite ongoing treatment may require high-intensity treatment (international normalized ratio, 3.0-4.0) with warfarin. 43 With regard to the management of renal artery stenosis, treatment with antihypertensive drugs, antiaggregation agents, and anticoagulants have been reported to be effective. Sangle et al41 analyzed 14 hypertensive APS patients with renal artery stenosis who received oral anticoagulation for more than 1 year and reported the beneficial effects of their approach on blood pressure control and renal function. The treatment for APSN is based on anticoagulation. Steroids, hydroxycloroquine, and some immunosuppressive agents occasionally have been reported to reduce the titer of aPL, but their role in reducing the risk of thrombosis has not yet been proven. A few studies evaluating the role of immunosuppressants on primary APSN have reported favorable effects.42,44 The long-term use of intravenous immunoglobulin also has been reported in very selected cases of primary relapsing APS despite standard treatment.45 New oral anticoagulants such as dabigatran, apixaban, and rivaroxaban now are available. They have been shown to be effective in the management of venous thromboembolism and they do not require laboratory monitoring. Preliminary experience supporting the use of new oral anticoagulants in patients with APS were reported recently. $46 \mathrm{~A}$ trial to assess the efficacy and safety of rivaroxaban in APS currently is underway.47 B-cell depletion with rituximab also has been suggested for selected cases of APS, mainly in the presence of severe thrombocytopenia.48 Recently, Erkan et al49 designed a 12-month, phase II pilot rituximab study, including adult aPL-positive patients with thrombocytopenia, cardiac valve disease, skin ulcer, aPL nephropathy, and/or cognitive dysfunction. A potential role of eculizumab, an anti-C5 antibody, in selected cases of APS also has been proposed. However, available evidence still is based on experimental APS models or anecdotal cases.50,51

\section{Take Home Messages}

The following points should be kept in mind. I

?] In patients affected by APS, both primary and associated with other autoimmune diseases such as SLE, the kidney is a major target organ. I 
[3 Renal involvement in APS is a well-recognized manifestation of the syndrome and is characterized by the occurrence of thrombosis, potentially involving any site of the kidney vasculature.

[? In SLE, APSN can co-exist with LN. A biopsy is mandatory for a differential diagnosis. I

[? Because of the clinical and histologic similarities between APSN and other microangiopathies, an aPL test is useful to distinguish between possible causes. I

2] The presence of Lupus Anticoagulant and/or aCL in SLE patients can be considered a risk factor for poor renal outcome even in the absence of $L N$. I

[? CAPS must be considered in the differential diagnosis of APSN because it is associated with high mortality and must be treated aggressively. I

?] During pregnancy the presence of aPL is associated with a higher risk of morbidity including hypertension, nephritis, preeclampsia, and eclampsia. I

? The use of hydroxychloroquine is recommended in patients with aPL associated with SLE even in the absence of APS.

[? Low-dose aspirin can be useful in patients with a higher risk of thrombosis (eg, triple positivity for aPL). I

[? Therapeutic approaches for renal involvement in APS focuses on anticoagulation with long-term warfarin. ।

[? Presently, there are not sufficient data regarding the use of new oral anticoagulants in patients with APSN. I

[? Rescue therapies, including intravenous immunoglobulins, rituximab, or eculizumab can be attempted in refractory cases.

REFERENCES 1. Miyakis S, Lockshin MD, Atsumi T, et al. International consensus statement on an update of the classification criteria for definite antiphospholipid syndrome (APS). J Thromb Haemost. 2006;4:295-306.

2. Worrall JG, Snaith ML, Batchelor JR, et al. SLE: a rheumatological view. Analysis of the clinical features, serology and immunogenetics of 100 SLE patients during long-term followup. QJM. 1990;74:319-30.

3. Estes $\mathrm{D}$, Christian $\mathrm{CL}$. The natural history of systemic lupus erythematosus by prospective analysis. Medicine (Baltimore). 1971;50:85-95.

4. Rovin BH, Parikh SV. Lupus nephritis: the evolving role of novel therapeutics. Am J Kidney Dis. 2014;63:677-90.

5. Ruiz-Irastorza G, Crowther M, Branch W, et al. Antiphospholipid syndrome. Lancet. 2010;376:1498-509.

6. Cervera R, Serrano R, Pons-Estel GJ, et al. Morbidity and mortality in the antiphospholipid syndrome during a 10-year period: a multicentre prospective study of 1000 patients. Ann Rheum Dis. 2015;74:1011-8. 7. Stone S, Pijnenborg R, Vercruysse L, et al. The placental bed in pregnancies complicated by primary antiphospholipid syndrome. Placenta. 2006;27:457-67. 
8. Cervera R, Tektonidou MG, Espinosa G, et al. Task Force on Catastrophic Antiphospholipid Syndrome (APS) and noncriteria APS manifestations (I): catastrophic APS, APS nephropathy and heart valve lesions. Lupus. 2011;20:165-73.

9. Stone S, Langford K, Nelson-Piercy C, et al. Antiphospholipid antibodies do not a syndrome make. Lupus. 2002;11:130-3.

10. Meroni PL, Borghi MO, Raschi E, et al. Pathogenesis of antiphospholipid syndrome: understanding the antibodies. Nat Rev Rheumatol. 2011;7:330-9.

11. Meroni PL, Riboldi R. Pathogenic mechanisms mediating antiphospholipid syndrome. Curr Opin Rheumatol. 2001;13:377-82.

12. Sciascia S, Cuadrado MJ, Khamashta M, et al. Renal involvement in antiphospholipid syndrome. Nat Rev Nephrol. 2014;10: 279-89.

13. Giannakopoulos B, Krilis SA. The pathogenesis of the antiphospholipid syndrome. N Engl J Med. 2013;368:1033-44.

14. Sciascia S, Sanna G, Murru V, et al. GAPSS: the Global AntiPhospholipid Syndrome Score. Rheumatology (Oxford). 2013; 52:1397-403.

15. Lie JT. Vasculitis in the antiphospholipid syndrome: culprit or consort? J Rheumatol. 1994;21:397-9.

16. Amigo MC, Garcia-Torres R, Robles M, et al. Renal involvement in primary antiphospholipid syndrome. J Rheumatol. 1992;19:1181-5.

17. Sinico RA, Cavazzana I, Nuzzo M, et al. Renal involvement in primary antiphospholipid syndrome: retrospective analysis of 160 patients. Clin J Am Soc Nephrol. 2010;5:1211-7.

18. Tektonidou MG, Sotsiou F, Nakopoulou L, et al. Antiphospholipid syndrome nephropathy in patients with systemic lupus erythematosus and antiphospholipid antibodies: prevalence, clinical associations, and long-term outcome. Arthritis Rheum. 2004;50:2569-79.

19. Daugas E, Nochy D, Huong DL, et al. Antiphospholipid syndrome nephropathy in systemic lupus erythematosus. J Am Soc Nephrol. 2002;13:42-52.

20. Uthman I, Khamashta M. Antiphospholipid syndrome and the kidneys. Semin Arthritis Rheum. 2006;35:360-7.

21. Griffiths MH, Papadaki L, Neild GH. The renal pathology of primary antiphospholipid syndrome: a distinctive form of endothelial injury. QJM. 2000;93:457-67.

22. Rankin EC, Nield GH, Isenberg DA. Deterioration of renal function in a patient with lupus. Ann Rheum Dis. 1994;53: 67-71.

23. Alchi B, Griffiths M, Jayne D. What nephrologists need to know about antiphospholipid syndrome. Nephrol Dial Transplant. 2010;25:3147-54.

24. Barbour T, Johnson S, Cohney S, et al. Thrombotic microangiopathy and associated renal disorders. Nephrol Dial Transplant. 2012;27:2673-85. 
25. Bertolaccini ML, Amengual O, Andreoli L, et al. 14th International Congress on Antiphospholipid Antibodies Task Force. Report on antiphospholipid syndrome laboratory diagnostics and trends. Autoimmun Rev. 2014;13:917-30.

26. Asherson RA, Espinosa G, Cervera R, et al. Catastrophic antiphospholipid syndrome: proposed guidelines for diagnosis and treatment. J Clin Rheumatol. 2002;8:157-65.

27. Nochy D, Daugas $E$, Droz $D$, et al. The intrarenal vascular lesions associated with primary antiphospholipid syndrome. J Am Soc Nephrol. 1999;10:507-18.

28. Bhandari S, Harnden P, Brownjohn AM, et al. Association of anticardiolipin antibodies with intraglomerular thrombi and renal dysfunction in lupus nephritis. QJM. 1998;91:401-9.

29. Zheng $\mathrm{H}$, Chen $\mathrm{Y}$, Ao $\mathrm{W}$, et al. Antiphospholipid antibody profiles in lupus nephritis with glomerular microthrombosis: a prospective study of 124 cases. Arthritis Res Ther. 2009;11: R93.

30. Miranda JM, Garcia-Torres R, Jara L, et al. Renal biopsy in systemic lupus erythematosus: significance of glomerular thrombosis. Analysis of 108 cases. Lupus. 1994;3:25-9.

31. Ruiz-Irastorza G, Egurbide MV, Ugalde J, et al. High impact of antiphospholipid syndrome on irreversible organ damage and survival of patients with systemic lupus erythematosus. Arch Intern Med. 2004;164:77-

82.

32. Moroni G, Ventura D, Riva P, et al. Antiphospholipid antibodies are associated with an increased risk for chronic renal insufficiency in patients with lupus nephritis. Am J Kidney Dis. 2004;43:28-36.

33. Pérez Velásquez C, Isenberg D, Croca S. Secondary antiphospholipid syndrome nephropathy and lupus nephritis: a casecontrol study [abstract THU0308]. Ann Rheum Dis. 2013;72 (Suppl 3):270.

34. Kwok LW, Tam LS, Zhu T, et al. Predictors of maternal and fetal outcomes in pregnancies of patients with systemic lupus erythematosus. Lupus. 2011;20:829-36.

35. Smyth A, Oliveira GH, Lahr BD, et al. A systematic review and meta-analysis of pregnancy outcomes in patients with systemic lupus erythematosus and lupus nephritis. Clin J Am Soc Nephrol. 2010;5:2060-8.

36. Kincaid-Smith P, Fairley KF, Kloss M. Lupus anticoagulant associated with renal thrombotic microangiopathy and pregnancy-related renal failure. QJM. 1988;68:795-815.

37. Kincaid-Smith P. Post partum renal failure in systemic lupus erythematosus associated with circulating lupus anticoagulant and anticardiolipin antibody. Clin Nephrol. 1991;36:52

38. Ruiz-Irastorza G, Cuadrado MJ, Ruiz-Arruza I, et al. Evidencebased recommendations for the prevention and long-term management of thrombosis in antiphospholipid antibodypositive patients: report of a task force at the 13th International Congress on antiphospholipid antibodies. Lupus. 2011;20: 206-18.

39. Erkan D, Aguiar CL, Andrade D, et al. 14th International Congress on Antiphospholipid Antibodies Task Force Report on Antiphospholipid Syndrome Treatment Trends. Autoimmun Rev. 2014;13:685-96.

40. Tektonidou MG, Laskari K, Panagiotakos DB, et al. Risk factors for thrombosis and primary thrombosis prevention in patients with systemic lupus erythematosus with or without antiphospholipid antibodies.

Arthritis Rheum. 2009;61:29-36. 
41. Sangle SR, D'Cruz DP, Abbs IC, et al. Renal artery stenosis in hypertensive patients with antiphospholipid (Hughes) syndrome: outcome following anticoagulation. Rheumatology (Oxford). 2005;44:372-7.

42. Korkmaz C, Kabukcuoğlu S, Isiksoy S, et al. Renal involvement in primary antiphospholipid syndrome and its response to immunosuppressive therapy. Lupus. 2003;12:760-5.

43. Brodsky SV, Nadasdy T, Rovin BH, et al. Warfarin-related nephropathy occurs in patients with and without chronic kidney disease and is associated with an increased mortality rate. Kidney Int. 2011;80:1819.

44. Sokunbi DO, Miller F, Wadhwa NK, et al. Reversible renal failure in the primary antiphospholipid syndrome-a report of two cases. J Am Soc Nephrol. 1993;4:28-35.

45. Sciascia S, Giachino O, Roccatello D. Prevention of thrombosis relapse in antiphospholipid syndrome patients refractory to conventional therapy using intravenous immunoglobulin. Clin Exp Rheumatol. 2012;30:409-13.

46. Sciascia S, Breen K, Hunt BJ. Rivaroxaban use in patients with antiphospholipid syndrome and previous venous thromboembolism. Blood Coagul Fibrinolysis. 2015;26:476-7.

47. Giles I, Khamashta M, D'Cruz D, et al. A new dawn of anticoagulation for patients with antiphospholipid syndrome? Lupus. 2012;21:1263-5.

48. Sciascia S, Naretto C, Rossi D, et al. Treatment-induced downregulation of antiphospholipid antibodies: effect of rituximab alone on clinical and laboratory features of antiphospholipid syndrome. Lupus. 2011;20:1106-8.

49. Erkan D, Vega J, Ramón G, et al. A pilot open-label phase II trial of rituximab for non-criteria manifestations of antiphospholipid syndrome. Arthritis Rheum. 2013;65:464-71.

50. Erkan D, Lockshin MD. New approaches for managing antiphospholipid syndrome. Nat Clin Pract Rheumatol. 2009;5:160-70.

51. Velik-Salchner C, Lederer W, Wiedermann F. Eculizumab and renal transplantation in a patient with catastrophic antiphospholipid syndrome: effect of heparin on complement activation. Lupus. 2011;20:772. 
Figure 1. APS-associated nephropathy. (A) Hematoxylin-eosin stain. Intracapillary thrombi in the glomerulus on the left. The afferent arteriole is occluded entirely by a fibrin thrombus (black arrow). (B) Same stain. Fibrinoid necrosis and mucoid intimal edema in the intima of an arteriole (red arrow) with fragmented red blood cells. The interstitium around the arteriole is edematous. (C) Same stain. Thrombi occluding peritubular capillaries (black arrow). Interstitial edema, hemorrhage, and acute tubular injury might be present depending on the severity of the acute ischemic injury. (D) Large intravascular thrombus at the vascular pole of a glomerulus is red under trichrome staining (black arrow). (E) Direct immunofluorescence showing positive staining for fibrinogen in glomerular capillary lumina and at the vascular pole (white arrows). (F) At ultrastructural examination fibrin occludes the capillary lumen. Endothelial cells have lost fenestration and podocytes show extensive foot process effacement. (G) In chronic disease, double contours without interposition of electron dense immune complexes can be seen (white arrow). Platelets are seen in capillary lumina in both acute and chronic phases (black circle). Podocytes often are injured and show foot process effacement (black arrows). Figure reprinted with permission from Sciascia et al.1
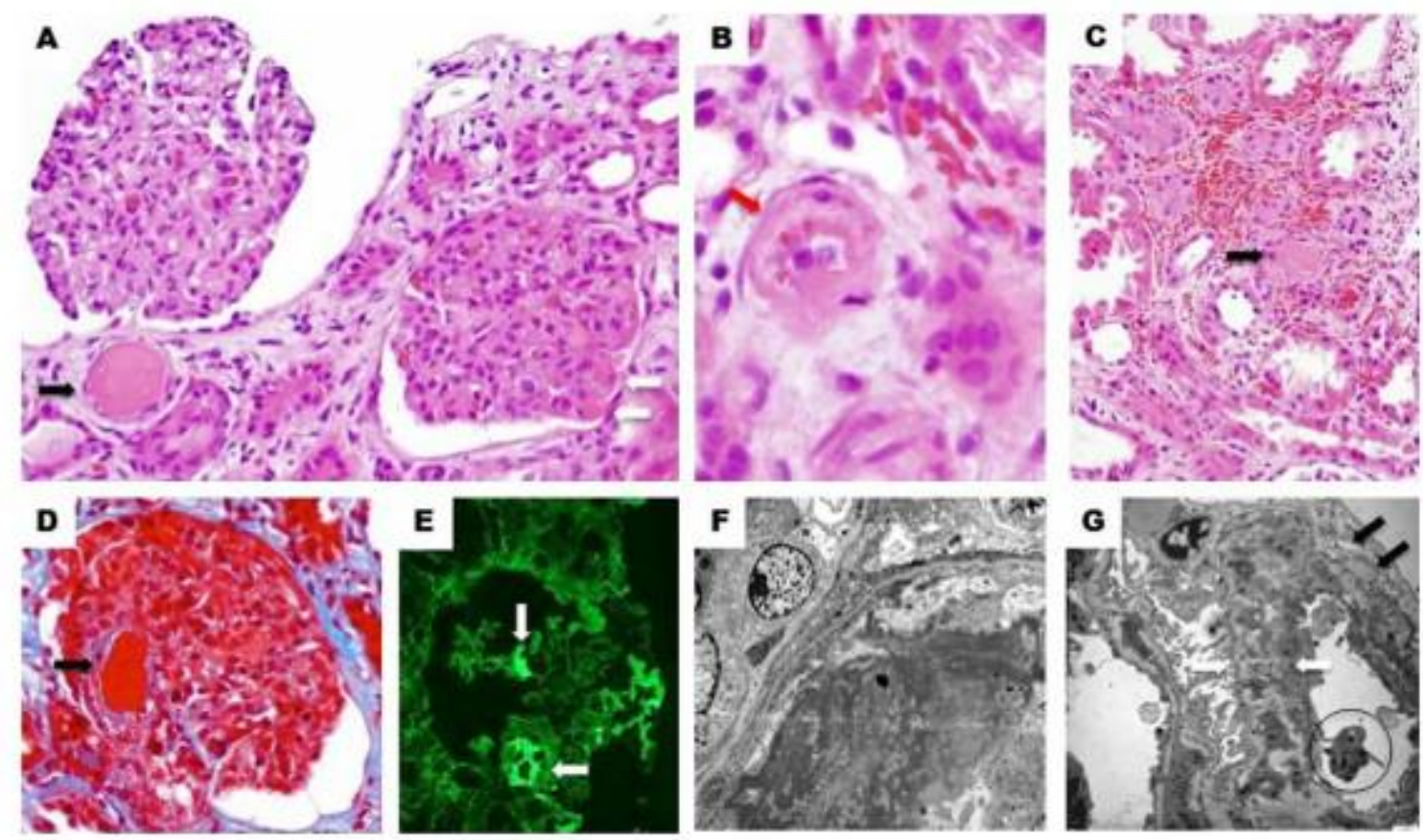
Table 1. Criteria for the Classification of Antiphospholipid Syndrome

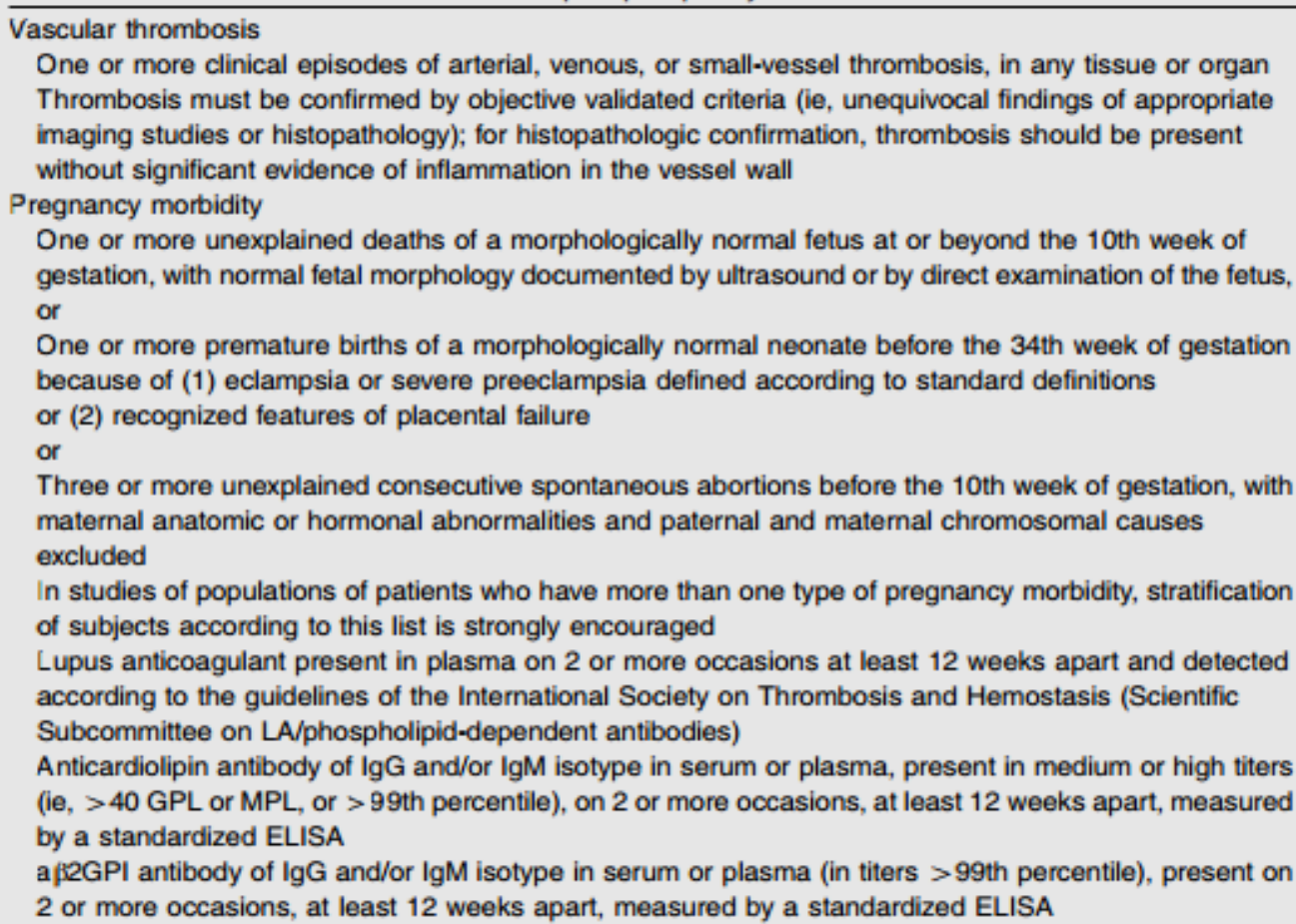

One or more clinical episodes of arterial, venous, or small-vessel thrombosis, in any tissue or organ Thrombosis must be confirmed by objective validated criteria (ie, unequivocal findings of appropriate imaging studies or histopathology); for histopathologic confirmation, thrombosis should be present without significant evidence of inflammation in the vessel wall

Pregnancy morbidity

One or more unexplained deaths of a morphologically normal fetus at or beyond the 10 th week of gestation, with normal fetal morphology documented by ultrasound or by direct examination of the fetus, or

One or more premature births of a morphologically normal neonate before the 34th week of gestation because of (1) eclampsia or severe preeclampsia defined according to standard definitions or (2) recognized features of placental failure

or

Three or more unexplained consecutive spontaneous abortions before the 10th week of gestation, with maternal anatomic or hormonal abnormalities and paternal and maternal chromosomal causes excluded

In studies of populations of patients who have more than one type of pregnancy morbidity, stratification of subjects according to this list is strongly encouraged

Lupus anticoagulant present in plasma on 2 or more occasions at least 12 weeks apart and detected according to the guidelines of the International Society on Thrombosis and Hemostasis (Scientific Subcommittee on LA/phospholipid-dependent antibodies)

Anticardiolipin antibody of $\mathrm{IgG}$ and/or IgM isotype in serum or plasma, present in medium or high titers (ie, > $40 \mathrm{GPL}$ or MPL, or >99th percentile), on 2 or more occasions, at least 12 weeks apart, measured by a standardized ELISA

api2GPI antibody of IgG and/or IgM isotype in serum or plasma (in titers >99th percentile), present on 2 or more occasions, at least 12 weeks apart, measured by a standardized ELISA

Definite antiphospholipid syndrome may be diagnosed if at least one of the clinical criteria and at least one of the laboratory criteria are met.

Abbreviation: ELSA, enzyme-linked immunosorbent assay; GPL, IIgG phospholipid units; MPL, IgM phospholipid units.

Table 2. Clinical Features of the Antiphospholipid Syndrome

Venous thrombosis: deep venous thrombosis

Arterial thrombosis: strokes, transient ischemic attacks, multi-infarct dementia, or myocardial infarction

Pregnancy complications: recurrent pregnancy loss, intrauterine growth restriction, pre-eclampsia

Thrombocytopenia

Leg ulcers, livedo reticularis, thrombophlebitis, and Sneddon's syndrome

Laboratory criteria

Budd-Chiari syndrome and pulmonary thromboembolism

Heart valve lesions

Transverse myelitis, chorea, and epilepsy

Hemolytic anemia, Coombs' positivity, and Evans' syndrome

Pulmonary hypertension

Cognitive impairment

Chronic headache

Splinter hemorrhages

Labile hypertension and accelerated atherosclerosis

Other less common Ischemic necrosis of bone

Bone marrow necrosis

Addison's disease

Guillain-Barré syndrome and pseudomultiple sclerosis

Amaurosis fugax

Sensorineural hearing loss

Renal artery and vein thrombosis and microangiopathy

Retinal artery and vein thrombosis

Digital gangrene

Table 3. Renal Manifestations of Antiphospholipid Syndrome

\author{
APS nephropathy \\ Renal artery stenosis and thrombosis \\ Renal vein thrombosis \\ Renal infarction \\ Systemic hypertension \\ aPL-related glomerular microthrombosis in lupus nephritis \\ Catastrophic antiphospholipid syndrome
}


Table 4. Therapeutic Recommendations in Individuals With Antiphospholpid Antbodies

Strict control of cardiovascular risk factors should be accomplished in all individuals with a high-risk aPL profle," irrespective of the presence of previous thrombosis, concomitant SLE, or additional APS features

All aPL carriers should receive thromboprophylaxis with the usual doses of LMWH in high-risk situations, such as surgery, prolonged immobilzation, and puerperium

In patients with SLE and posifive Iupus anticoagulant or isolated persistent anticardiolipin antibodies at medium-high titers, primary thromboprophylaxis with hydroxyehloroquine \pm low-dose aspirin is recommended

In non-SLE individuals with aPL and no previous thrombosis, long-term primary thromboprophylaxis with low-dose aspirin is recommended in patients with a high-risk aPL profle, especially in the presence of other thrombofic risk factors

Patients with either arterial or venous thrombosis and aPL who do not fulfil criteria for APS should be trealed in the same manner as aPL-negative patients with similar thrombotic events

Patients with definite APS and a first venous evert should receive oral antioosgulant therapy to a target INA of 2.0 to 3.0

Patients with definite APS and arterial thromboeis should be treated with warfarin at an INR $>3.0$ or combined anti-aggregant-anticoagulant therapy (INR 2.0-3.0)

The patient's bleeding risk should be estimated before prescribing high-intensity anticoagulant or combined anti-aggregant-anticoagulant therapy

Non-SLE patients with a first noncardioembolic cerebral arterial event, with a low-risk aPL profile and the presence of reversible trigger factors could be considered individual candidates for treatment with antiplatelet agents

Duration of treatment

Indefinite antithrombotic therapy is recommended in pafents with definite APS and frombosis

In cases of first venous event, low-risk aPL profle ${ }^{\dagger}$ and a known transient precipitating factor, anticoagulation could be limited to $3-6$ months

In patients with difficult management owing to recurrent thrombosis, fluctuating INR levels, major bleeding or at a high risk for major bleeding, alternative therapies could include long-term LMWH. hydroxychloroquine, or statins APS with poor obstetric outcomes

Recurrent earty (pre-embryonic or embryonic) miscarriage Low-dose aspirin alone, or plus:

LMWH at thromboprophylactic doses

Fetal death ( $>10$ weeks' gestation) or prior early delivery ( $<34$ weeks of gestation) caused by severe preeclampsia or placental insufficiency

Low-dose aspirin, plus:

LMWH at thromboprophylactic doses

Women with previous thrombotic APS during pregnancy

Low-dose aspirin, plus:

LMWH

INR, international normalized ratio, LMWH, low-molecular-weight heparin.

•High-risk APL profile: lupus anticosgulant posifvity, triple positivity (lupus anticoagulant + anticardiolpin + ap2GPI antibodies). isolated persistently positive anticardiolipin antibodies at medium-high titers.

${ }^{\dagger}$ Low-risk aPL profile: isolated, intermittently posifive anticardiolipin or ant-B2-ghycoprotein I at low-medium fiters.

Primary thromboprophylaxis in SLE patients with antiphospholipid antibodies

Primary

thromboprophylaxis in aPL-positive individuals without SLE

Secondary

thromboprophylaxis

Refractory and diflicut

cases

Antiphospholipid syndrome in pregnancy 